\title{
Leggere le figure, vedere i testi, riflettere le parole. Strategie di analisi del testo letterario e produzione scritta in Italiano L3
}

\author{
Margherita MESIRCA \\ École Européenne Bruxelles II
}

\begin{abstract}
Questo contributo si propone di condividere i risultati di un percorso di Ricerca Azione svolto nell'ambito del Master di Secondo Livello in Progettazione avanzata dell'insegnamento della lingua e cultura italiana a stranieri (Itals) dell'Università di Venezia. Il percorso - svoltosi nell'ambito della mia attività didattica di docente di Italiano L3 alla Scuola Europea di Bruxelles II - è stato dedicato a valutare l'efficacia di un'analisi del testo letterario affine a quella solitamente proposta in Italiano LI, a partire dall'ipotesi che la qualità approfondita dell'analisi potenziasse la produzione scritta, ossia mettesse in atto un processo virtuoso di "trascinamento" della qualità della lingua scritta ad essa sottesa.
\end{abstract}

Keywords: italiano L3, analisi del testo letterario, figure retoriche, fluency, produzione scritta.

Da docente di Latino, Italiano Li e Italiano L3 alla Scuola Europea di Bruxelles $\mathrm{II}^{\mathrm{I}}$ ho avuto l'opportunità di insegnare parallelamente Italiano Lingua I e Lingua 3 a due classi che ho accompagnato dalla $\mathrm{S}_{4}$ alla $\mathrm{S}_{7}$ (corrispondenti rispettivamente alla prima liceo e all'anno di maturità). Questa esperienza mi ha permesso di riflettere a lungo - e sul campo - attorno ai processi di apprendimento nella Li e nella L3, su affinità e differenze, portandomi ad approfondire alcuni aspetti relativi all'intensificazione delle competenze in Italiano L3, in particolare la produzione scritta.

Nel curriculum delle Scuole Europee lo studio di una L3 è obbligatorio per tutti gli allievi dal primo al quinto anno della scuola secondaria e opzionale nel sesto e settimo

Il Programma della Lingua 3 si fonda sul Quadro comune europeo di riferimento per l'insegnamento e l'apprendimento delle lingue straniere e sul Quadro europeo delle competenze chiave per l'istruzione e la formazione permanente ${ }^{2}$. Il programma della Lingua 3 per il terzo ciclo - quello relativo agli ultimi due

\footnotetext{
I Le Scuole Europee sono scuole intergovernative create in sinergia tra l'Unione europea e i Governi degli Stati membri; lo stato giuridico di cui godono in ciascun paese è quello di istituzioni di diritto pubblico. Rivolte principalmente ai figli del personale delle Istituzioni Europee, le Scuole Europee hanno come vocazione quella di fornire un insegnamento multilingue e multiculturale dalla scuola materna alla scuola superiore. Attualmente ne esistono I3, sono ripartite in 6 paesi (Germania, Italia, Belgio, Spagna, Olanda e Lussemburgo) e accolgono più di 27.000 alunni.

2 https://www.coe.int/en/web/common-european-framework-reference-languages.
} 
anni di scuola secondaria - prevede che l'allievo debba essere in grado di «leggere e capire testi letterari e non letterari e saper scrivere testi dettagliati [...] che esprimano punti di vista o impressioni su una larga gamma di argomenti».

All'interno delle linee guida per l'insegnamento della L3 contenute nel programma, quattro sono i concetti chiave: la variazione del peso delle abilità nei cicli, e la conseguente centralità della scrittura nell'ultimo; il concetto di "effetto terza lingua", ossia l'opportuno ricorso ad abilità linguistiche già acquisite; la centralità della differenziazione; la peculiarità dei gruppi di L3, dato che spesso implicano la compresenza di competenze molto diverse, da quella del principiante assoluto a quella del bilingue. Secondo le Linee guida, la differenziazione può essere realizzata nelle strategie di insegnamento e di apprendimento, nelle difficoltà dei testi e delle attività proposti.

Relativamente all'abilità di "Leggere, comprendere e analizzare testi letterari e non letterari nel terzo ciclo", il programma specifica che «per analisi deve intendersi la capacità di esprimere il messaggio principale del testo, aiutandosi, se necessario, con alcuni termini tecnici di base».

Attorno a una peculiare forma di differenziazione e a un'estensione del concetto di analisi del testo rispetto alla definizione presente nel programma si è basato il percorso di RA ${ }^{3}$ cui ho dedicato la tesi del Master di Secondo livello in Progettazione avanzata dell'insegnamento della lingua e della cultura italiane a stranieri. A partire dalla richiesta "paradossale" presente nel programma di Italiano L3 - ossia quella di proporre due libri da leggere uguali per tutti gli studenti nelle classi S6 e in S7 (penultimo e ultimo anno), da analizzare in modo semplice, al contempo differenziando le attività all'interno del percorso di lettura - la mia risposta "paradossale" è stata quella di proporre un'analisi del testo che non si limitasse (come suggerito dal programma) a indagare il messaggio principale del testo, ma che si configurasse come possibilità di andare a fondo di tale messaggio, nel dettaglio, nell'implicito, attraverso le figure retoriche, da proporre con domande aperte, con l'intento di scoprire se in un tale percorso ogni studente potesse trovare il suo spazio di risposta, di riflessione, di espressione, di approfondimento.

All'interno di quanto previsto dal programma di Italiano L3 - ossia la lettura obbligatoria di due libri, uno in S6 e uno in $\mathrm{S}_{7}$, uguali per tutte le Scuole Europee - ho cercato quindi di costruire nel biennio 2017-2019, un percorso di lettura che permettesse al contempo di approfondire e di differenziare. Durante l'anno scolastico 20I7-I8, con la classe S6 abbiamo letto

3 Come scrive Maria De Luchi, un progetto di Ricerca-Azione è un progetto in cui il docente «assume lo status di ricercatore» (20I6: 23); la teoria della Ricerca-Azione, che nasce dalle intuizioni di Kurt Lewin (1946), «vede il docente come protagonista principale del processo di ricognizione, analisi, raccolta dei dati e riflessione sul suo operato» (De Luchi 20I6: 23). Sulla Ricerca-Azione si vedano, tra gli altri, Burns (1999), Trombetta - Rosiello (2000), Barbier (2007). 
Wok di Francesco Carofiglio e in maniera naturale (per la mia formazione) ho proposto agli studenti una modalità di analisi del testo affine a quella utilizzata con gli studenti di italiano Lr: figure retoriche, temi, significati impliciti, reticoli simbolici, proponendo compiti e attività anche complessi. Il risultato è stato molto positivo: forte coinvolgimento, miglioramento della qualità della produzione scritta, trascinamento della L3 verso alcuni tratti qualitativi della Li - nello specifico relativi alla raffinatezza delle idee.

Per l'anno scolastico 20I9-20 il libro da leggere è stato Dove troverete un altro padre come il mio di Rossana Campo. Visto che - come il percorso su Wok aveva mostrato - l'accesso ai significati impliciti di un testo letterario, attivando l'immaginario e costruendosi come percorso progressivo e condiviso, aveva permesso un'intensificazione della qualità della riflessione e della produzione scritta - intensificazione che avevo rilevato soggettivamente (coinvolgimento degli studenti, qualità dei dibattiti e delle prove scritte, richiesta di leggere ancora "come l'anno scorso") - mi sono quindi riproposta di verificare la possibilità di progettare un percorso di analisi che permettesse di misurare e monitorare in maniera strutturata e progressiva la qualità della competenza nella produzione scritta (e quindi della riflessione) attraverso l'analisi dettagliata della qualità delle risposte degli allievi agli esercizi di produzione e analisi proposti durante la lettura del libro.

Se il percorso dedicato a Wok aveva mostrato che la qualità delle competenze si costruisce nella condivisione sistematica del testo letterario attraverso attività approfondite di comprensione, analisi, riflessione e produzione scritta, nella RA dedicata al testo di Rossana Campo mi sono proposta di valutare l'efficacia di un percorso focalizzato sulle figure retoriche, in quanto peculiari strumenti di analisi che permettono di vedere i testi, e quindi di vedere oltre le parole - ossia di lavorare attorno ai significati impliciti - e dunque di raffinare la riflessione, e perciò di potenziare la produzione scritta. L'intento è stato cioè quello di monitorare il processo attraverso il quale la qualità approfondita della riflessione/visione del testo indotta dalla messa a fuoco delle figure ha un effetto di virtuoso trascinamento della qualità della scrittura negli esercizi di analisi approfondita e commento del testo.

Al contempo ho inteso verificare la potenzialità delle figure retoriche come strumenti di differenziazione all'interno di un percorso di lettura. Le figure retoriche sono infatti immagini inscritte in un testo, che irradiano significato attraverso il loro effetto (Barthes I980: 130); dato che le domande sulle figure che ho proposto negli esercizi di produzione sono al contempo chiuse e aperte, ossia partono da un elemento testuale vincolante e univoco, che però è per sua natura portatore della densità del significato della letteratura (Barthes i980: I30; Ricoeur I975: 87) e in quanto tale è passibile di diversi livelli di analisi, riflessione e approfondimento, questo ha permesso a ciascuno studente di raffinare la produzione e l'analisi a partire dal suo livello di competenza. 


\section{Esercizi per trasformare la conoscenza}

Le schede proposte durante questo percorso, relative all'analisi del testo a partire da una riflessione sulla funzione e sull'effetto di una o più figure in un singolo segmento (Fase I), sulla funzione e sull'effetto di una o più figure a partire dal segmento per approdare all'argomento o al tema implicato (Fase 2), sulla funzione e sull'effetto di una o più figure a partire dal segmento per approdare a tutto il testo (Fase 3), si inscrivono in quella che Bereiter e Scardamalia (1987: 87) definiscono «knowledge trasforming strategy». Quast'ultima, a differenza dell'altra strategia efficace per sviluppare la composizione scritta - ossia la «knowledge telling strategy» - richiede di adattare la conoscenza per creare nuovi contenuti ${ }^{4}$, e quindi, nel caso specifico di questi esercizi, di interagire con il codice retorico imposto dal dominio dell'analisi del testo.

\section{Le figure come immagini e lo sviluppo dell'expertise}

Per la loro peculiare richiesta di trasformare la conoscenza, gli esercizi sulle figure retoriche proposti nel percorso implicano di coordinare spiegazione, argomentazione, sintesi, e in questo senso inducono a sviluppare quella che Alamargot definisce expertise rédactionelle.

Secondo l'autore, per indurre gli studenti a produrre testi più elaborati e fluidi, si può o isolare una componente del processo compositivo ed esercitarla, oppure migliorare la coordinazione tra i diversi momenti del processo compositivo (Alamargot et al. 2010: 25; Alamargot-Morin-Drijbooms 2016: 47). Secondo Alamargot è difficile insegnare o esercitare tale coordinazione, che è una competenza essenziale senza la quale non c'è sviluppo di quella che l'autore definisce appunto expertise rédactionelle (Alamargot 2018: 5) 5 .

Le riflessioni di Bereiter e Scardamalia sulla complexity of skills come elemento specifico della expertise mostrano come i concetti di complessità del processo e controllo delle parti del processo siano fondamentali (Bereiter-Scardamalia 1986: I2). Nel caso di questi esercizi lo studente-esperto deve esercitare quelle che Widdowson definisce procedural abilities, ossia quelle procedure interpretative necessarie a interpretare il testo letterario (Widdowson 1986: 136$)^{6}$.

4 Sull'interpretazione come dimensione più elevata e complessa della comprensione cfr. anche Perkins 2007.

5 Bereiter e Scardamalia, definiscono l'expertise «high levels of knowledge and skill that do not come about naturally but require a special and sustained investment in learning» (1986: IO). 6 A tal proposito e in connessione con la rilevanza del lavoro interpretativo a partire dal testo letterario, Widdowson afferma come «with literary discourse the actual procedures for making sense are much more in evidence. You've got to employ interpretative procedures in a way which isn't requires of you in the normal reading process. If you want to develop these 
Gli esercizi sulle figure richiedono infatti di coordinare un processo complesso e di esercitare uno specifico controllo dettato dal tempo a disposizione e dalla formulazione sintetica della risposta. Il processo di produzione sotteso agli esercizi proposti nel corso della RA ha richiesto di padroneggiare le seguenti dimensioni:

a) sintesi - a diversi livelli: si parte da un segmento del testo (Fase I) per andare al collegamento tra la figura e l'argomento o il personaggio (Fase 2) e infine il collegamento richiesto è tra la figura e l'intero testo (Fase 3);

b) spiegazione: viene chiesto di mettere a fuoco la funzione e l'effetto della figura;

c) argomentazione: il collegamento richiesto necessità di essere supportato da argomenti testuali.

Gli esercizi sulle figure, in altre parole, hanno permesso di praticare quella che Perkins, sulla scia di Duffy-Jonassen (1992), definisce performance perspective, secondo la quale «understanding a topic of study is a matter of being able to perform in a variety of thought-demanding ways with the topic, for instance to: explain, muster evidence, find examples, generalize, apply concepts, analogize, represent in a new way» (Perkins I993: 5).

\section{Effetti delle figure retoriche nel processo della lettura}

La riflessione sulle figure retoriche potenzia il processo di lettura. L'assestamento progressivo del reticolo di significati che dà origine secondo Kintsch (1998: 953) alla base del testo nel processo di lettura trova infatti un suo potenziamento nel parallelo processo di assestamento del reticolo retorico. È come se il testo procedesse secondo un doppio reticolo: quello delle figure è il reticolo visivo ${ }^{7}$.

Se - come sostengono Miall e Kuiken - gli aspetti stilistici dei testi emergono dal testo inducendo nel lettore un effetto di defamiliarizzazione e la reazione emotiva del lettore alle componenti formali dei testi è particolarmente intensa nei confronti di quelli defamiliarizzanti, che tendono a sfidare la sua cornice cognitiva (Miall-Kuiken I994: 389), il reticolo retorico si costruisce a sua volta in questa dialettica tra ciò che è familiare e ciò che è straniante, tra immagine sfocata e immagine nitida; il processo del reticolo retorico va dalla sfocatura delle immagini singole alla nitidezza delle immagini intrecciate dall'intero sistema testuale.

procedural abilities to make sense of discourse, then literature has a place» (1986: I36).

7 In questo senso il reticolo retorico rappresenta una sorta di dimensione implicita di quella che Caon e Spaliviero definiscono «semiosi complessa» dei testi letterari (2015: 26), ossia l'interazione tra la parte linguistica e la componente visiva. 
Le figure possono essere infatti considerate delle immagini implicate nel testo $^{8}$, o meglio delle implicature visibili ${ }^{9}$. Come sostiene Semino (2008: Io) infatti, l'utilizzo delle metafore viene spiegato non solo ricorrendo a termini come «image schemata», ma anche «scenes» (Grady I997 : passim) o «scenarios» (Musollf 2004: passim); il processo che porta alla visibilità di queste «scene» viene inscritto nel testo dal peculiare funzionamento delle figure retoriche, legato appunto al meccanismo testuale dell'implicatura.

Secondo Sbisà (2009: ad vocem) «l'implicatura si presenta come un alone di senso non ben definito, rispetto a cui ciascuna singola esplicitazione è in qualche modo manchevole (troppo precisa e insieme non esauriente), e può avere anch'essa, in certi casi, carattere sfumato e aperto». Ne consegue checontinua Sbisà - se «cogliere la presenza di implicature e regolarsi di conseguenza è possibile anche senza giungere a una esplicitazione», quest'ultima, tuttavia, «aggiunge la piena accessibilità e manipolabilità dell'implicito» (Sbisà 2009: ad vocem). Come scrive Iser (1972: 28I), infatti, le implicature, nel venire elaborate dal lettore, conferiscono al testo un significato molto più denso.

Il gioco che lo scrittore propone al lettore è quello di cogliere il significato implicito delle figure di cui costella il testo da un lato e, contemporaneamente, la specificità di quell'implicito rispetto al sistema del testo. Questo reticolo di significazioni implicite viene inoltre arricchito e reso denso dal fatto che la specificità dell'implicito delle figure in un testo si inscrive nella sua letterarietà, ossia nella rielaborazione delle figure attuata dallo scrittore.

Secondo la teoria della metafora concettuale di Lakoff e Johnson (I980), infatti, se la metafora come elaborazione della mente permea la nostra vita quotidiana, la creatività metaforica nasce dal processo cognitivo sotteso alla trasformazione dei contenuti concettuali delle metafore stesse ${ }^{\mathrm{I}}$. Perelman e Olbrechts-Tyteca rilevano, d'altro canto, che la forza di un'espressione metaforica «viene contemporaneamente dalla familiarità che si ha con essa e dalla conoscenza abbastanza imprecisa dell'analogia che ne costituisce l'origine» $(1966: 430)^{11}$.

Di conseguenza, l'esercizio sulle figure è contemporaneamente un esercizio sull'implicito e un esercizio sul sistema del testo, in quanto implica un

8 Sulla comprensione da parte dei lettori delle metafore dal punto di vista dell'immagine cfr. Gibbs-Bogdonovich 1999; sulla metafora come oggetto di inferenze su tipi complessi di implicazioni cfr. Gibbs (1999: 268-270).

9 I paragoni metaforici sono, secondo Glucksberg e Keysar (1993), delle affermazioni implicite di inclusione in classi, ossia di appartenenza a nuove categorie degli oggetti dissimili, che mettono in connessione.

Io A tal proposito Cristina Cacciari sottolinea come le espressioni metaforiche siano il riflesso di processi cognitivi radicati nell'esperienza (I991: 218).

II Sull'interazione tra parole e pensiero cfr., tra gli altri, Vygotskij (2002, passim). 
doppio livello di riflessione: che cosa significa questa figura? Che cosa significa in relazione al resto del testo? Quale il suo effetto derivante dal contesto testuale in cui è inscritta?

Se consideriamo, inoltre, che la metafora non risiede tanto nelle parole, quanto nelle idee (Cacciari I99I: 2; Lakoff I99I, passim) e che dunque, come sostiene Pinker, le metafore letterarie inducono a risolvere l'incongruità di cui sono portatrici attraverso la messa a fuoco di una somiglianza inedita (Pinker 2007: 264), sono quindi proprio gli aspetti metaforici della lingua a rivelare la struttura poetica della mente (Gibbs 2005:3) e, allo stesso tempo, sono le figure retoriche a fornire al lettore «instruments for establishing imaginative connections between diverse emotions, ideas, and events» (Gibbs I996: 26), ossia gli strumenti per pensare per immagini.

Le implicazioni del comprendere un testo per via metaforica e quindi immaginativa sono piuttosto ricche, dato che, da un punto di vista pedagogico, si ritiene che un supporto immaginativo rappresenti un aiuto alla comprensione del testo poiché - provocando quella che Harris definisce absorption - poiché (Harris 2000: 342) facilita la modellizzazione della situazione e la comprensione tra gli avvenimenti (Glenberg-Langston I992: I4O-4I; Oostendorp-Goldman 1999: 85). Secondo Alamargot, inoltre, scrivere un testo a partire da un'immagine facilita il processo di pianificazione e alimenta il contenuto del testo (Alamargot et al. 20II: 507-508; Dansac-Alamargot 1999: 90). Questo significa che lavorare sul reticolo retorico di un testo permette non solo di "pensare per immagini", ma di "scrivere per immagini", ossia di intensificare la scrittura grazie alla comprensione immaginativa, operando secondo quello che Turner definisce metaphoric blend (Turner 2002: Io), ossia secondo l'operazione concettuale consistente nel «combining two mental packets of meaning - two schematic frames of knowledge or two scenarios, for example selectively and under constraints to create a third mental packet of meaning that has new, emergent meaning». Di conseguenza, lavorare sul reticolo retorico rappresenta un'opportunità di apprendimento legata a quello che definisce cognitive engagement; quest'ultimo, se massimizzato, è una garanzia, secondo Cummins, di academical progress (I979: IO).

Il processo del metaphoric blend viene inoltre potenziato dalle emozioni, dato che, come evidenzia Longo (20II: 2IO), la processazione di immagini mentali implica quello che viene definito come emotional arousal ${ }^{12}$. Questo

I2 Sull'interazione tra metafora, emozione ed immaginazione cfr. anche Sadoski-Paivio (200I: 29I-292). Sulla funzione di potenziamento dell'apprendimento propria delle figure retoriche cfr. Pugh-Hicks-Davis (1997). Sul rapporto tra cognizione ed emozione cfr. l'interessante capitolo VI di Schumann (1999). Sulle aree di sovrapponibilità tra studi sulle emozioni e studi sull'immaginario letterario, quali il legame tra processi cognitivi e memoria associativa, supremazia dell'immaginazione, mimesi come simulazione cfr. Tan (2000: II8-I25). Sull'eccezionalità delle emozioni cfr. Barbero 2010: 32; sul potenziamneto del pensiero come piacere intellettuale cfr. Balboni 2006: Io. 
significa che leggere e scrivere attorno alle metafore potenzia il pensiero, l'immaginazione e l'emozione, che a loro volta intensificano la lettura e, come vedremo, la scrittura ${ }^{13}$.

\section{Articolazione del percorso}

Il percorso di RA è durato tre mesi, si è articolato in tre fasi, nelle quali sono stati svolti quarantotto esercizi di analisi e riflessione sulle figure retoriche del testo, per un totale di circa sessanta pagine scritte da ciascuno studente. A queste vanno aggiunti cinque questionari e una verifica finale. I dati pertinenti e rilevanti (Altricher-Posch-Someckh in Losito-Pozzo, 2005: I73-I74, in De Luchi 2007: 39) sono stati le risposte scritte alle domande, le risposte ai questionari, la verifica finale, il diario dell'insegnante. A parte quest'ultimo, tutte le altre schede con le domande e le risposte sono state i dati primari (De Luchi 2007: 28) della RA; con i termini usati da Woods (in De Luchi 2007: 29) si tratta di «compiti e risposte ai compiti».

I criteri di valutazione delle risposte sono stati i seguenti:

I) Efficacia alta: si legge con agio e si apprezza la risposta anche a prescindere dalla correttezza

2) Profondità = rilevazione dell'implicito

Profondità alta $=$ presenza di riflessioni sull'implicito

3) Originalità = presenza di collegamenti personali

Originalità alta $=$ articolazione di collegamenti personali

4) Qualità alta dei collegamenti con l'argomento in questione: ha un effetto di reciprocità. Il collegamento serve per mettere a fuoco la figura, la figura serve per mettere a fuoco l'argomento.

5) Fluency P (Presente) = concetto che comprende le caratteristiche di velocità di composizione, quantità e qualità del testo, organizzazione delle idee

I "numeri" finali della RA, ossia le percentuali ottenute dalla media delle ricorrenze dei valori alti attribuiti da me e da Giovanni Massidda - il collega di filosofia che con grande acutezza ed efficacia mi ha coadiuvata nella triangolazione - ai parametri considerati nella valutazione delle schede, per ciascuna fase della RA sono i seguenti:

I. l'efficacia è cresciuta dalla fase I alla fase 3 , in cui ha toccato il suo picco, per scendere lievemente nella verifica finale (87/95/93/93);

2. la profondità è decisamente aumentata rispetto alla fase I in tutte le altre fasi, con un picco nella fase 2, e un leggero aumento nella verifica finale rispetto alla fase $3(79 / 98 / 90 / 93)$;

I3 Sul potere euristico della metafora cfr., tra gli altri, Black (1979), Ortony (1975), Ricoeur (I975); quest'ultimo parla, a proposito della metafora, di «heuristique de la pensée» (1975: 32). 
3. l'originalità ha avuto un netto incremento nelle fasi 2 e 3 rispetto alla I, mentre si è assestata, nella verifica finale, ai valori di partenza $(70,90,88$, 70);

4. la qualità dei collegamenti è molto alta nelle fasi 2 e 3, ed è diminuita nella verifica finale $(83,84,76)$.

Alla luce di questi dati è possibile affermare che la crescente complessità degli esercizi ha supportato l'efficacia delle risposte; ha permesso la fluency per tutti gli studenti; ha supportato la profondità delle risposte; ha supportato l'originalità delle risposte; ha supportato la qualità dei collegamenti; ha toccato il suo livello più alto nella verifica finale, che si è rivelata un compito molto impegnativo dal punto di vista dell'originalità e dei collegamenti, richiedendo di mettere a fuoco l'interazione tra le figure più ricorrenti e il loro rapporto con le emozioni rappresentate nel testo e suscitate nel lettore; ha cioè richiesto non solo la padronanza di tutto il testo come la Fase 3, ma del reticolo retorico operante lungo tutto il testo e simultaneamente.

\section{Organizzazione e interpretazione dei dati nella FASE I}

La prima sequenza di esercizi ha richiesto di commentare l'effetto e la funzione delle figure retoriche nel segmento testuale proposto. Il questionario finale della FASE I ha messo in luce come la difficoltà degli esercizi li abbia fatti rientrare in quella che Vygotskij definisce "zona di sviluppo prossimale", ossia «la distanza tra il livello attuale di sviluppo come determinato dal problem solving individuale e il livello di sviluppo come determinato dal problem solving sotto la guida di un adulto» (Vygotskij I987:34). Gli studenti hanno infatti trovato la difficoltà degli esercizi tra normale e abbastanza elevata e hanno dichiarato - contemporaneamente - di avere, mediamente, una capacità abbastanza elevata di analisi e commento delle figure retoriche. Rispetto agli esercizi svolti nel percorso dedicato alla lettura di Wok, gli esercizi sulle figure retoriche dedicati a Dove troverete un altro padre come il mio si sono rivelati diversi e sono stati percepiti come particolarmente efficaci per la comprensione del testo (gli studenti hanno definito mediamente abbastanza elevata la maggior comprensione del testo dopo gli esercizi). Tra gli elementi in comune troviamo infatti ricorrenti la rilevazione della sorprendente compatibilità tra accessibilità dell'esercizio e complessità delle figure; la percezione della natura aperta delle domande e della possibile qualità non esaustiva delle risposte; la consapevolezza della funzione delle figure per la visualizzazione e immaginazione del testo e per l'accesso alla sua profondità.

\section{Organizzazione e interpretazione dei dati nella FASE 2}


Stanti i risultati dell'analisi e interpretazione dei dati della Fase I, gli obiettivi della Fase 2 sono stati da un lato valutare la tenuta della qualità delle risposte (efficacia, profondità, originalità) nonostante l'intensificazione della complessità dei quesiti; di conseguenza valutare l'efficacia degli esercizi come strumento per intensificare la qualità della scrittura e quindi, contestualmente, anche misurare l'efficacia degli esercizi dal punto di vista della differenziazione anche nel momento in cui la richiesta si fa più complessa. Nella seconda fase della RA, partendo dalla maggior comprensione del testo acquisita dagli studenti grazie agli esercizi della prima fase, ossia dall'intensificazione che si è prodotta grazie ad essi, si è deciso di intensificare a sua volta la difficoltà, passando alla richiesta di commentare la funzione e l'effetto delle figure retoriche non più solo relativamente al segmento testuale in questione, ma anche rispetto all'argomento o al tema implicati nel segmento stesso (come, per esempio, la relazione tra due personaggi); e quindi di sfruttare, attraverso questa estensione, il processo di significazione implicita delle figure a livello di quello che Todorov definisce simbolismo proposizionale (Todorov I978: 609).

L'analisi e l'interpretazione dei dati attuate nel monitoraggio e alla fine della Fase 2 ci hanno visti concordi nell'affermare come gli esercizi abbiano continuato a riflettere il paradosso della complessità: la qualità della produzione scritta di tutti gli studenti, come interazione dei parametri efficacia/ originalità/profondità - ha continuato infatti ad assestarsi tra alta o medio/ alta nonostante l'intensificazione della complessità degli esercizi. L'analisi e l'interpretazione del questionario finale della Fase 2, le cui risposte hanno tutte un punteggio medio di 3 , corrispondente a "abbastanza elevato/a" - ha permesso di mettere in luce lo specifico articolarsi del paradosso della complessità. Gli studenti, infatti, hanno continuato a percepire il proprio senso di autoefficacia, ritenendo abbastanza elevata la qualità delle loro risposte; si sono al contempo rivelati consapevoli dell'intensificazione della complessità, giudicando abbastanza elevata la differenza di difficoltà tra gli esercizi della prima fase e della seconda. La maggiore complessità si è rivelata compatibile con la qualità della scrittura utilizzata (giudicata abbastanza elevata dagli studenti), dell'analisi e del ragionamento, valutati altrettanto abbastanza elevati qualitativamente. Gli esercizi hanno inoltre intensificato la dimensione cognitiva, come evidenziato dalla capacità degli studenti di spiegare in modo articolato il processo mentale attraverso cui li hanno svolti. 


\section{Organizzazione e interpretazione dei dati nella FASE 3}

Stanti i risultati della FASE 2, gli obiettivi della FASE 3 sono stati quelli di valutare la capacità degli studenti di articolare in Italiano L3 l'intero reticolo retorico-tematico del testo - ossia di passare dalle figure proposte ai nodi semantici impliciti in esse inscritti dall'intero sistema retorico del testo - e, di conseguenza, di ri-tracciare i nuclei tematici irradiati dalle figure; di riflettere e far riflettere gli studenti sulla differenza tra la stessa risposta in L3 e in LI o L2 e quindi valutare la specificità cognitiva degli esercizi di analisi del testo a partire dalle figure retoriche; di valutare la tenuta della qualità delle risposte in termini di profondità, originalità ed efficacia, per tutti gli studenti, nonostante $\mathrm{o}$, paradossalmente, grazie alla maggior complessità richiesta.

Per la valutazione delle schede nella Fase 3, ai criteri di efficacia, profondità e originalità sono stati aggiunti:

\section{QUALITÀ DEL RETICOLO RETORICO/TEMATICO MESSO A FUOCO:}

$\mathrm{ALTA}=\mathrm{il} \mathrm{reticolo} \mathrm{è} \mathrm{a} \mathrm{più} \mathrm{nodi/temi}$

MEDIA = il reticolo è composto di due temi o figure, ossia quella del testo e un'altra

$\mathrm{BASSA}=$ il reticolo è solo accennato

\section{DIFFERENZA CONTENUTI LI/L2/L3}

ALTA: Nel testo in LI/L2 ci sono almeno 2 argomenti in più MEDIA: Nel testo in LI/L2 c'è almeno un argomento in più BASSA: Il testo è equivalente dal punto di vista delle idee

Data la natura degli esercizi della Fase 3 che richiedevano, in un tempo breve e in un testo breve, di condensare delle risposte a domande complesse sulla globalità del testo a partire dalla specificità del suo reticolo retorico, ho ritenuto opportuno introdurre anche il criterio della FLUENCY, concetto che comprende le caratteristiche di velocità di composizione, quantità e qualità del testo, organizzazione delle idee e conoscenza delle convenzioni di scrittura nella lingua di destinazione $\mathrm{I}^{\mathrm{I}}$.

L'analisi e l'interpretazione dei dati attuate nel monitoraggio e alla fine della FASE 3 ci hanno visti concordi nell'affermare che la qualità della produzione scritta di tutti gli studenti, come interazione dei parametri efficacia/ originalità/profondità/fluency/qualità dei collegamenti - ha continuato ad assestarsi tra alta o medio/alta nonostante l'intensificazione della complessità degli esercizi. Il paradosso della complessità ha permesso agli studenti di muoversi con padronanza all'interno del testo e nell'utilizzare il reticolo retorico come telaio con cui tirarne i fili.

I4 La definizione è di Hester (200I) e viene ripresa in Abdel Latif (20I2). 


\section{Un percorso tra il paradosso della complessità e il paradosso dell'originalità}

L'analisi e l'interpretazione dei dati hanno permesso di rilevare che tutti gli studenti hanno trovato nel percorso di lettura il loro spazio di risposta, di riflessione, di approfondimento, di empatia ${ }^{15}$, di percezione del senso e dell'utilità della sua articolazione secondo la logica che Krashen definisce «the Pleasure Hypothesis» (2006: 2). Il corrispettivo di questa esperienza di lettura qualitativamente più raffinata è stata l'intensificazione della produzione scritta di tutti gli studenti in termini di efficacia, profondità, originalità e fluency.

La compresenza e l'interazione tra differenziazione e intensificazione si è inscritta ed è stata resa possibile dai due paradossi intrinseci alla natura specifica delle figure retoriche e al loro interagire sistemico all'interno del testo letterario: il paradosso della complessità e il paradosso dell'originalità.

Il primo paradosso ha permesso di intensificare la difficoltà degli esercizi senza che questa diventasse un ostacolo per gli studenti, né in termini di efficacia, né in termini di profondità, né in termini di originalità. Viceversa, l'intensificazione ha reso la complessità il fattore di attivazione dell'efficacia, della profondità e dell'originalità come forme di progressiva expertise nella produzione, nell'analisi e nella conoscenza del testo.

Il paradosso dell'originalità si inscrive nella natura peculiare delle domande insite negli esercizi proposti, domande, al contempo chiuse e aperte, come, metaforicamente, è chiuso e aperto il significato e la funzione di una figura retorica: a richieste molto puntuali e vincolanti, su segmenti specifici, gli studenti hanno sempre dato risposte diverse le une dalle altre e tendenzialmente molto originali. La figura retorica infatti è sempre univoca, ma il significato irradiato nel testo, interagendo con quello delle altre figure, dà vita a una dimensione testuale e semantica ricchissima di sfumature e riverberi. Dentro queste sfumature e riverberi si inserisce la singola sensibilità del lettore, che con il suo carico di emozioni e inclinazioni cognitive percorre il reticolo secondo traiettorie sempre diverse, e al contempo, sempre fondate testualmente.

I5 Sul ruolo dell'empatia nella lettura cfr. Oatley (1994: 57-59); sulla risposta del lettore al testo cfr. Balboni 2006, Barbero 20I0, Bertoni 1996, Keen 2007, Levorato 2000, Pezzini 2007. 


\section{Bibliografia}

Abdel Latif, Muhammad M. M., Recent Develpments in Writing Fluency Measurement, in Exloring EFL Fluency in Asia, New York, Palgrave MacMillan, 20I2, pp. 196-209.

Alamargot, Denis, Comment emmener les élèves à produire des textes, CNESCO, 20I8, Écrire et rédiger: comment guider les élèves dans leurs apprentissages. Notes des experts. https://www.cnesco.fr/fr/ecrire-et-rediger/.

Alamargot, Denis et al., «What Makes a Skilled Writer? Working Memory and Audience Awareness during Text Composition», Learning and individual differences, XXI, 5, 20II, pp. 505-5I6.

Alamargot, Denis - Morin, Marie France - Drijbooms, Elise, «Planificateur ou formulateur? Mise en évidence de deux stratégies de rédaction à partir d'un support imagé chez des élèves de CEI e de CM2», Pratiques, I73I74, 20I7, pp. I-I8.

Altrichter, Herbert - Posch.Peter - Somekh, Bridget, Teacher investigate their work, London, Routledge, 1993

Balboni, Paolo Emilio, Insegnare la letteratura italiana a stranieri. Risorse per docenti di italiano come lingua straniera, Roma, Guerra, 2006.

Barbero, Carola, Chi ha paura di Mister Hyde. Oggetti fittizi, emozioni reali, Genova, Melangolo, 2010.

Barbier, René, La ricerca-azione, Roma, Armando Editore, 2007.

Barthes, Roland, La retorica antica, Milano, Bompiani, 1980.

Bereiter, Carl - Scardamalia, Marlene, The Psychology of Written Composition, New York, Routledge, 1987.

Bertoni, Federico, Il testo a quattro mani. Per una teoria della lettura, Firenze, La Nuova Italia, 1996.

Black, Max, «How Metaphor work», Critical Inquiry, VI, I, I979, pp. I3I-I43.

Burns, Anne, Collaborative Action Research for English Language Teachers, Cambridge, Cambridge University Press, I999.

Cacciari, Cristina, «La metafora: da evento del linguaggio a struttura del pensiero», in C. Cacciari (a cura di), Teorie della metafora. L'acquisizione, la comprensione e l'uso del linguaggio figurato, Milano, Cortina, I99I, pp. I-3I.

Caon, Fabio - Spaliviero, Camilla, Educazione linguistica, letteraria, interculturale: intersezioni, Torino, Loescher/Bonacci, 2015.

Consiglio d'Europa, Quadro comune europeo di riferimento per le lingue: apprendimento, insegnamento, valutazione, Firenze, La Nuova Italia - Oxford, 2002.

Cummins, Jean, «Cognitive/Academic Language Proficiency, Linguistic Interdependence, the Optimum Age Question and Some Other Matters», Working Papers on Bilingualism, 19, 1979, pp. 199-205.

Dansac, Christophe - Alamargot, Denis, «Accessing referential information during text composition: when and why?», in Torrance Mark - Galbraith, 
David (eds), Knowing what to write: Cognitive processes in the generation, selection and development of ideas during text production, Amsterdam, Amsterdam University Press, 1999, pp. 79-97.

De Luchi, Maria, «Il docente ricercatore», Bollettino Itals, XIV, 66, 20I6, pp. 2I-29.

—. «Metodologia della ricerca nella didattica delle lingue», Bollettino Itals, 2007, pp. I-5I.

Jonassen, David M, Duffy, Thomas M., Constructivism: New Implications for Instructional Technology, Hillsdale, NJ, LEA Erlbaum Publishers, 1992.

Gibbs, Raimond W., Intentions in the Experience of Meaning, Cambridge-New York, Cambridge University Press, 1999.

- La poetica della mente. Pensiero, linguaggio e comprensione figurati, Viterbo, Sette Città, 2005.

Gibbs, Raimond W., «Metaphor as a Constraint on Text Understanding», in Britton, Bruce K. - Graesser, Arthur. C. (eds), Models of Understanding Text, Mahwah, NJ, LEA Erlbaum Publishers, I996, pp. 2I5-240.

Gibbs, Raimond W., Jr. - Colston, Herbert L., «Figurative language», in Traxler, Matthew J. - Morton, Ann - Gernsbacher (eds), Handbook of Psycholinguistics, second revised edition, Amsterdam, Academic Press Inc., 2006.

Gibbs, Raimond W. - Bogdanovich, Josephine, «Mental Imagery in Interpreting Poetic Metaphor», Metaphor and Symbol, XIV, I, I999, pp. 37-44.

Glenberg, Arthur M. - Langston, William E., «Comprehension of illustrated text: Pictures help to build mental models», Journal of Memory and Language, XXXI, 2, I992, pp. I29-I5I.

Glucksberg, Sam, Understanding Figurative Language. From Metaphors to Idiom, Oxford-New York, Oxford University Press, 200 I.

Glucksberg, Sam - Keysar, Boaz, «How metaphor works», in Ortony, Andrew (ed.), Metaphor and Thought, Cambridge, Cambridge University, I993, pp. 404-424.

Grady, Joseph, Foundations of Meaning: Primary Metaphors and Primary Scenes, Dissertation, University of California, Berkeley, I997, https:/escholarship.org/uc/item/3g9427m2.

Harris, Paul L., The work of the imagination, Malden, MA, Blackwell Publishers, 2000.

Hester, John L., «Investigating writing fluency in seventh and eight graders' narrative and expository first drafts», JDAI-A, LXII, 3, 200I, pp. 884-90I.

Iser, Wolfgang, «The reading process: a phenomenological approach», New Literary History, III, 2, 1972, pp. 279-289.

Keen, Susanne, Empathy and the novel, Oxford, Oxford University Press, 2007.

Kintsch, Walter, Comprehension: A paradigm for Cognition, Cambridge, Cambridge University Press, I998. 
Kovecses, Zoltan, Metaphor: A practical introduction, Oxford, Oxford University Press, 2002.

Krashen, Stephen, «Pleasure Reading», Yourn Lerners Sig, Spring Issue, 2006, pp. 2-4.

Lakoff, George - Johnson, Mark, Metaphors We Live By, Chicago, University of Chicago Press, 1980.

Levorato, Maria Crstina, Le emozioni della lettura, Bologna, Il Mulino, 2000.

Lewin, Kurt, "Action Research and Minority Problems», Journal of Social Issues, II 4, 1946.

Longo, Giuseppe, Cognizione ed emozione. Processi di interpretazione del testo letterario dalle neuroscienze cognitive all'educazione emotiva, Lecce, Pensa Multimedia, 201 .

Losito, Bruno-Pozzo, Gabriella, La ricerca azione, Roma, Carocci, 2005.

Miall, Davis S. - Kuicken, Don, «Foregrounding, Defamiliarization, and Affect Response to Literary Stories», Poetics, XXII, 5, I994, pp. 389-407.

Musolff, Andreas, Metaphor and Political Discourse. Analogical Reasoning in Debates about Europe, Palgrave, Macmillan, 2004.

Oatley, Keith, «A Taxonomy of the Emotions of Literary Response and a Theory of identification in fictional narratives», Poetics, XXIII, I-2, I994, pp. 53-74.

Oostendorp, Herrev - Goldman, Susan R., The Construction of Mental Representations During Reading, Mahwah, Erlbaum, 1999.

Ortony, Andrew, «Why Metaphors are necessary and not just nice», Educational Theory, XXV, I, I975, pp. 45-53.

Perelman, Chaim - Olbrechts-Tyteca, L., Trattato dell'argomentazione, trad. it, Torino, Einaudi, 1966.

Perkins, David, «Teaching for Understanding», The professional journal of the American Federation of Teachers, XVII, 3, 1993, pp. 8-35.

- Making Learning Whole. How Seven Principles of Teaching Can Transform Education, San Francisco, Jossey-Bass, 2007.

Pezzini, Isabella, Il testo galeotto. La lettura come pratica efficace, Roma, Meltemi, 2007.

Pinker, Steven, The Stuff of Thought. Language as a Window into Human Nature, New York, NY, Viking, 2007.

Programma per tutte le lingue III. Rif.:20Io-D-49-it-6, reperibile sul sito www. eursc.org.

Pugh, Sharon - Hicks, Jean - Davis, Marcia, Metaphorical ways of Knowing. The Imaginative Nature of Thought and Expressions, Urbana, Illinois, National Council of Theachers of English, I997.

Ricoeur, Paul, La métaphore vive, Paris, Seuil, 1975.

Sadoski, Mark - Paivio, Allan, Imagery and Text. A Dual Coding Theory of Reading and Writing, Mahwah, Erlbaum, 200I. 
Sbisà, Marina, «L'implicito: forme e funzioni», in Enciclopedia Treccani, 2009, ad vocem.

Schumann, John, The Neurobiology of Affect in Language, Oxford, Blackwell, 1999.

Semino, Elena, Metaphor in Discourse, Cambridge, New York, Cambridge University Press, 2008.

Tan, Ed S., Emotion, Art and the Humanities, in Feldmann Barrett Lisa - Lewis, Michael - Haviland-Jones, Jeannette M., (eds), Handbook of Emotions, New-York-London, Guildford Press, 2000, pp. II6-I34.

Todorov, Tzvetan, Symbolisme et interprétation, Paris, Seuil, 1978.

Trinchero, Roberto, Manuale di ricerca educativa, Milano, Franco Angeli, 2002. Trombetta, Carlo - Rosiello, Loredana, La ricerca-azione. Il modello di Kurt Lewin e le sue applicazioni, Trento, Erickson, 2000.

Turner, Mark, «The Cognitive Study of Art, Language and Literature», Poetics Today, XXIII, I, 2002, pp. 9-20.

Vygotsky, Lev, Pensiero e linguaggio, Milano, Giunti, 2002.

-.Il processo cognitivo, Torino, Bollati Boringhieri, 1987.

Widdowson, Henry, «The Untrodden Ways», in Brumfit, Christopher -Carter, Ronald (eds), Literature and Language Teaching, Oxford University Press, 1986, pp. I33-I40.

Wolff, Francis, «Trois techniques de vérité dans la Grèce classique», Argumentation et Rhétorique I et II, Hermés, I5-I6, 1995, pp. 4I-7I. 